\title{
Soft Computing Techniques for Mutual Coupling Reduction in Metamaterial Antenna Array
}

\author{
Balamati Choudhury, Sangeetha Manickam, and R. M. Jha \\ Centre for Electromagnetics, CSIR-National Aerospace Laboratories, Bangalore 560017, India \\ Correspondence should be addressed to Balamati Choudhury; balamati@nal.res.in
}

Received 28 June 2013; Revised 24 September 2013; Accepted 25 September 2013

Academic Editor: Ling Wang

Copyright ( 2013 Balamati Choudhury et al. This is an open access article distributed under the Creative Commons Attribution License, which permits unrestricted use, distribution, and reproduction in any medium, provided the original work is properly cited.

Application of soft computing techniques for various metamaterial designs and optimizations is an emerging field in the microwave regime. In this paper, a global optimization technique, namely, particle swarm optimization (PSO), is used for the design and optimization of a square split ring resonator (SSRR) having a resonant frequency of $2.4 \mathrm{GHz}$. The PSO optimizer yields the structural parameters, which is further simulated and validated with the optimized value. This optimized structure results in the mutual coupling reduction in a microstrip antenna array designed for wireless application.

\section{Introduction}

Soft computing techniques play important role in the design and optimization in various electromagnetic applications including antenna engineering. The aim of these techniques is to tolerate imprecision and uncertainties and yet yield quick solutions that are both robust and economically viable. Recent developments in the rapidly growing field of metamaterials have caught the attention of researchers worldwide due to its intrinsic properties leading to exciting applications in engineering such as the invisibility cloak, high-performance frequency selective surfaces (FSS) and radomes, and so forth. An important aspect of these metamaterial applications is the design and optimization towards actual hardware realization for which soft computing techniques have emerged as a potential tool due to their promise to treat the enormous complexity in analysis with relative ease [1].

Microstrip patch antennas are widely used in wireless communication in various platforms including aerospace due to their features like compactness, compatibility with MMIC (conformal) design, and high performance. In practice, mutual coupling effects which degrade the antenna performance are predominant [2]. Such mutual coupling effects are often mitigated by the use of EBG structures, which however makes the antenna array system very complex [3]. In order to overcome this complexity, a simple square split ring resonator (SSRR) is proposed in this paper. Such an application requires design of metamaterial structures at a desired frequency range (equivalent to the resonant frequency of the antenna array, mutual coupling of which is to be reduced). A particle swarm optimization (PSO) algorithm is developed here, which optimizes the resonant frequency and provides the structural parameters. This optimized design is further used for mutual coupling reduction and performance enhancement of the antennas.

In this paper, an array of simple single ring SSRR is used for reduction of mutual coupling in a microstrip patch antenna array, which is designed at $2.36 \mathrm{GHz}$ for wireless applications. The PSO algorithm optimizes this SSRR structure. A single array of optimized SSRR is then placed over a microstrip antenna array, and the mutual coupling effect is studied for different positions of the SSRR array.

\section{PSO Design for SSRR}

Soft computing techniques are essentially a collection of computational techniques in computer science, artificial intelligence, and machine learning. Thus there are many techniques available under this unique umbrella terminology such as artificial neural network (ANN), genetic algorithm (GA), fuzzy logic (FL), particle swarm optimization (PSO), bacteria foraging optimization (BFO), and so forth [4]. 


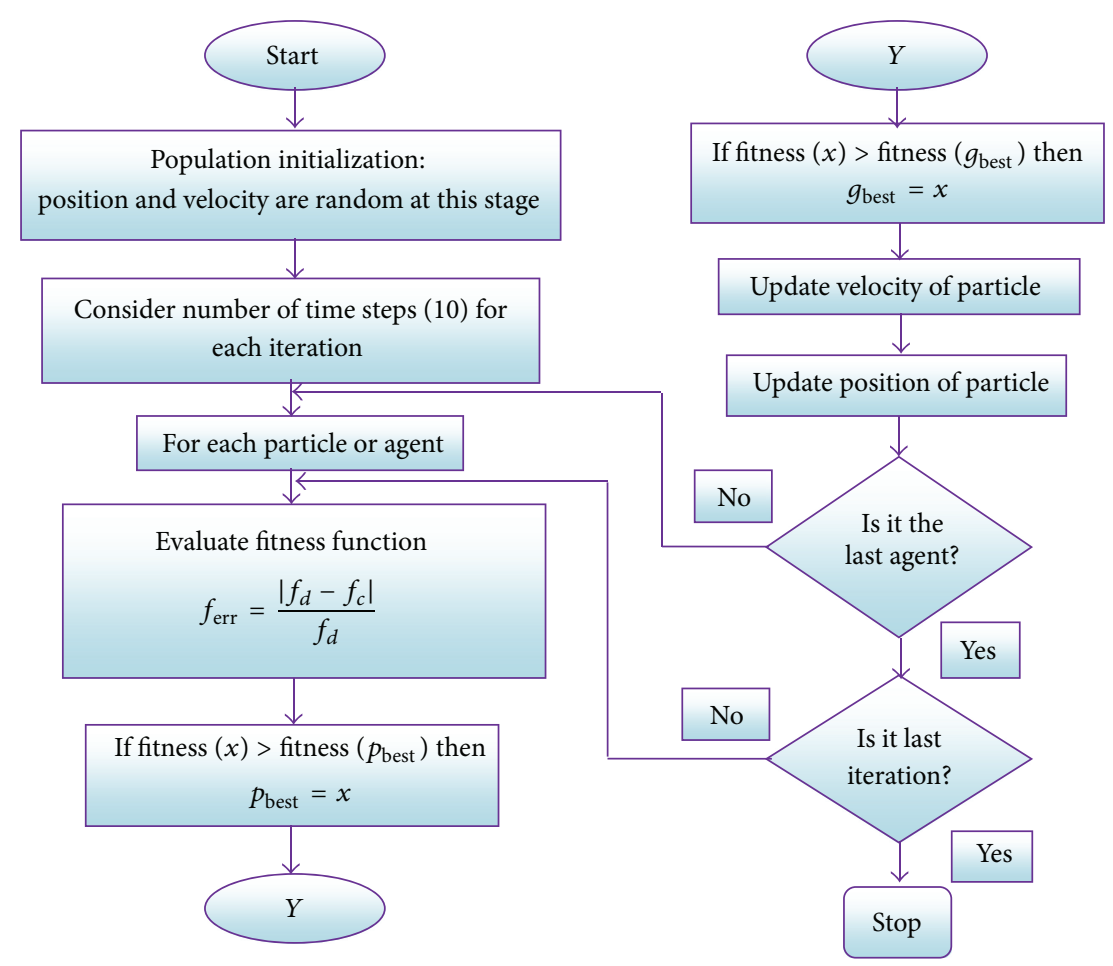

FIGURE 1: Flowchart of the proposed PSO optimization problem.

As it is apparent from the terminology, many of the soft computing techniques are inspired from biological phenomena and the social behavior of biological populations. Likewise, the recently developed methods based on swarm intelligence and foraging behavior of natural and biological populations (such as birds, fish, ants, and even bacteria) too are considered to be part of the growing field of soft computing [5-7].

Among the techniques available for soft computing, the PSO is one of the global optimization methods based on the movement and intelligence of swarms, which optimizes difficult multidimensional discontinuous problems. It is an evolutionary computational technique proposed by Kennedy and Eberhart in 1995 [6]. The PSO is a promising simple yet effective and robust method used for search and optimization in diverse electromagnetic problems. The development of PSO can be illustrated through an analogy similar to a swarm of bees in a field. The goal of a swarm of bees in a field is to find the location with the highest density of flowers. This motivates the engineers to use PSO as an optimization technique. The flowchart of the basic PSO algorithm implemented for the proposed optimization problem is shown in Figure 1.

\section{Implementation of PSO}

SSRR is a metamaterial structure which consists of single square shaped ring with gap. This structure is printed on a dielectric substrate of thickness $0.6 \mathrm{~mm}$ and permittivity 4.4. The schematic of an SSRR with the dimensions is shown in Figure 2, where "l" denotes the side length of the SSRR, " $w$ " denotes the width of conductor, and " $g$ " denotes the gap present in the rings. The equivalent circuit of the square SRR is a parallel $L C$ tank circuit, given in Figure 1. The resonant frequency of the square SRR is obtained by equivalent circuit analysis method. In this method, the distributed network is converted to lumped network (Figures 2(b) and 2(c)) and analysis is carried out [8]. by

The resonant frequency of the split ring resonator is given

$$
f_{r}=\frac{1}{2 \pi \sqrt{L C}}
$$

where $L$ is total inductance and $C$ is gap capacitance which is dependent on structural parameters of SSRR [9]. The expressions for $L$ and $C$ are given below:

$$
L=2 L_{1}+L_{2}+2 L_{3}, \quad C=\frac{K c t \varepsilon_{0}}{g},
$$

where $K$ is the correction factor, $\varepsilon_{0}$ is the free-space permittivity, $c$ is the velocity of light, $t$ is the thickness of SSRR, and $g$ is the gap spacing in the SSRR.

The PSO optimizer acts here as a CAD package, which yields the structural parameters such as the length, width, and spacing for a desired resonant frequency.

The fitness function used for this optimization is

$$
f_{\text {err }}=\frac{\left|f_{d}-f_{c}\right|}{f_{d}}
$$

where $f_{d}$ is the desired frequency and $f_{c}$ is the frequency obtained using the equivalent circuit analysis. The PSO optimizes the fitness function and extracts the structural 




(a)

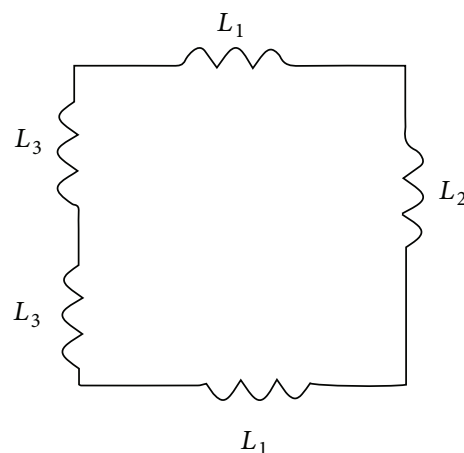

(b)



(c)

FIgURE 2: (a) Top view of a unit cell of the metamaterial periodic structure. (b) Equivalent inductance of the SSRR. (c) Equivalent circuit of the SSRR.



(a)

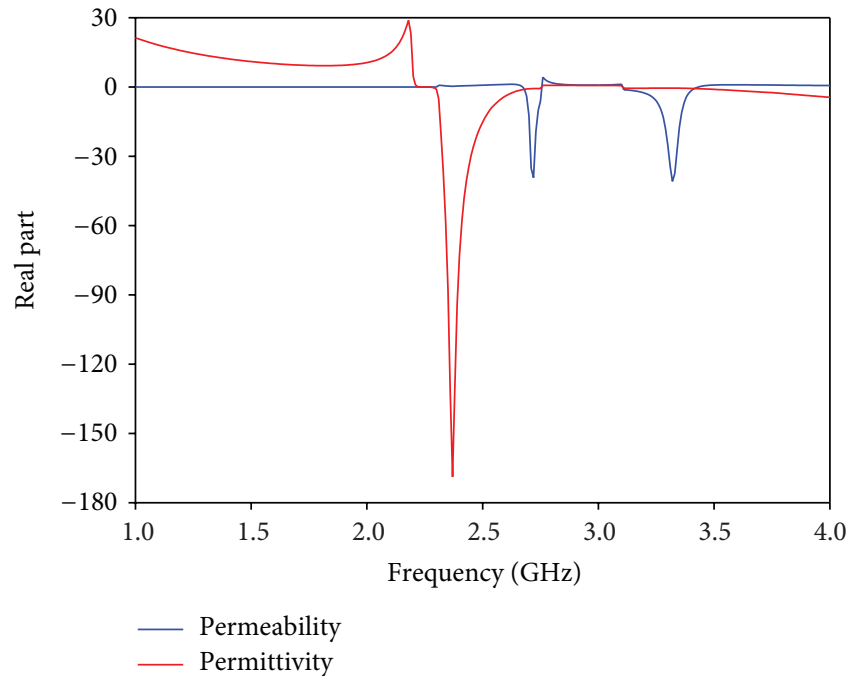

(b)

FIGURE 3: (a) Scattering parameters $S_{11}$ and $S_{21}$ of the designed SSRR using PSO. (b) Extracted permittivity and permeability of the designed SSRR using PSO.

parameters. The extracted length $l$, width $w$, and the gap spacing $g$ are $9 \mathrm{~mm}, 0.18 \mathrm{~mm}$, and $0.125 \mathrm{~mm}$, respectively. These optimized values are used for design of the SSRR. The boundary conditions were assigned accordingly during the design of SSRR [10]. The permittivity and permeability values are extracted using (5) [11]. The simulated scattering parameters $\left(S_{11}\right.$ and $\left.S_{21}\right)$ and the corresponding extracted permittivity and permeability are given in Figures 3(a) and $3(\mathrm{~b})$. The metamaterial characteristics of the proposed structure are readily inferred in Figure 3(b), from 2.3 to $2.6 \mathrm{GHz}$. Consider

$$
\begin{gathered}
n=\frac{1}{k d} \cos ^{-1}\left[\frac{1}{2 S_{21}}\left(1-S_{11}^{2}+S_{21}^{2}\right)\right], \\
z=\sqrt{\frac{\left(1+S_{11}^{2}\right)^{2}-S_{21}^{2}}{\left(1-S_{11}^{2}\right)^{2}-S_{21}^{2}}},
\end{gathered}
$$

$$
\begin{aligned}
\varepsilon & =\frac{n}{z}, \\
\mu & =n z,
\end{aligned}
$$

where $w$ is the radian frequency and $d$ is the thickness of the unit cell.

The PSO algorithm is implemented to optimize the structural SSRR parameters such as width, length, and gap between the slits, for a desired frequency of operation with the overall objective to reduce the mutual coupling in the antenna array.

Two different algorithms, namely, PSO and bacteria foraging optimization (BFO), have been implemented towards optimization of square SRR. For this optimization problem, the details of accuracy and execution time for both the algorithms are given in Table 1 . The design parameters considered are substrate dielectric constant 3.86 and desired frequency is 




(a)

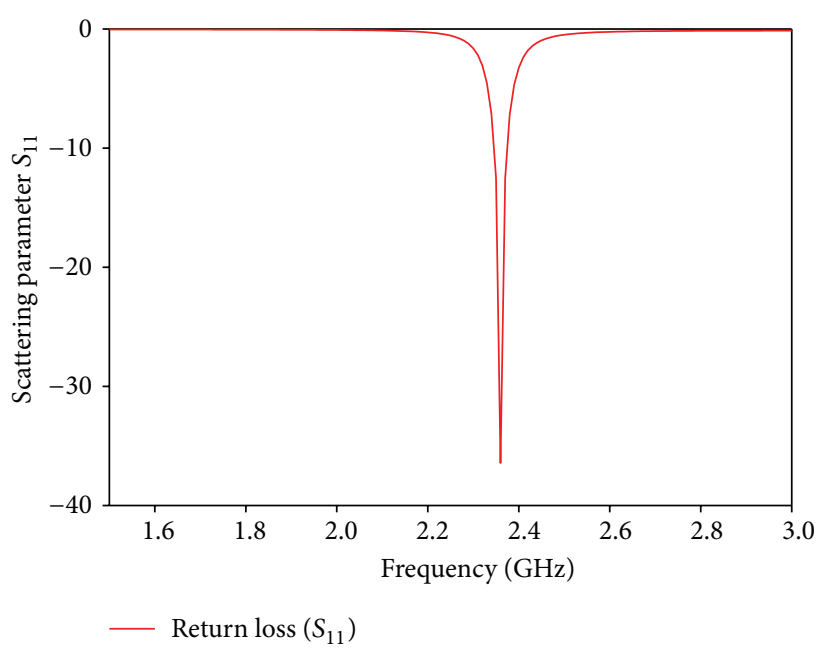

(b)

FIGURE 4: (a) Schematic of the microstrip antenna array and (b) return loss of the antenna array.

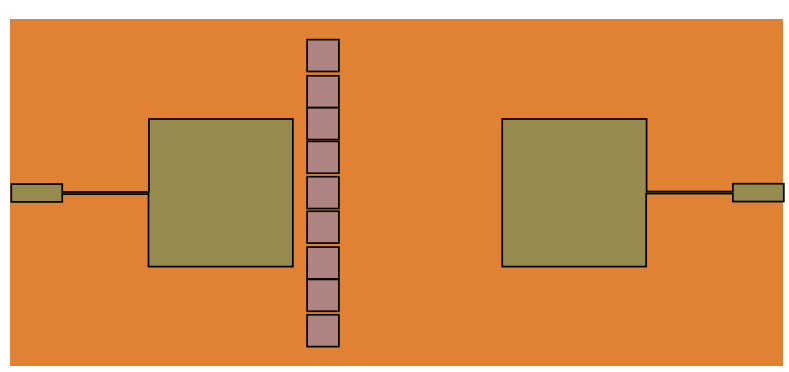

(a)

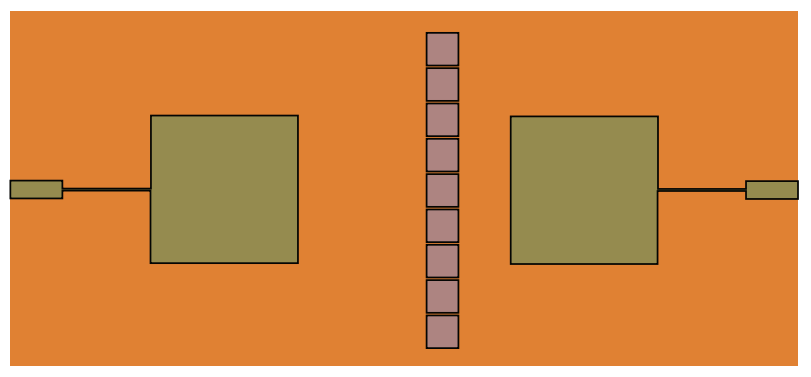

(c)

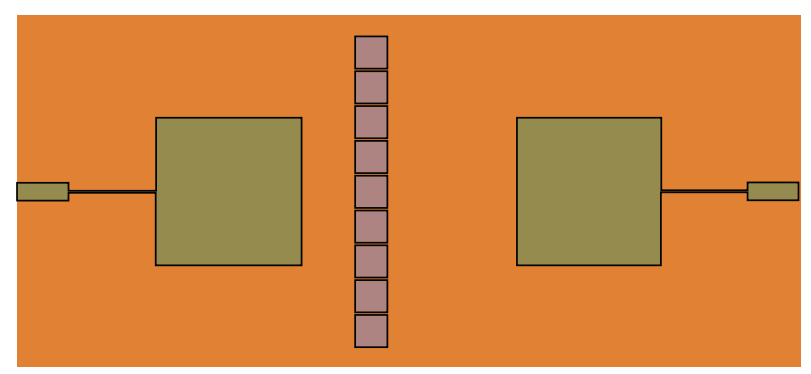

(b)

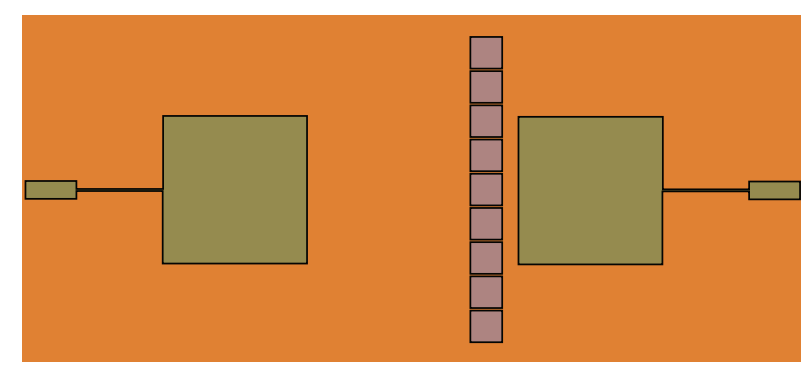

(d)

FIGURE 5: An array of SSRR loaded in between the antenna elements. (a) Position 1, (b) position 2, (c) position 3, and (d) position 4.

2.36 GHz. The computation is done using an Intel Dual-core 4 GB RAM PC.

It is readily observed that for the class of problem in hand, PSO is more accurate and highly convergent compared to the BFO. Hence PSO algorithm is considered in this work, for mutual coupling reduction in antenna array design.

\section{Geometry of the Antenna Array}

Microstrip antennas are best suited and are widely used for commercial and wireless applications. In this work, a simple microstrip array of two elements is designed having operating frequency $2.36 \mathrm{GHz}$. The microstrip array antenna is designed on a Duroid substrate with dimension $220 \mathrm{~mm} \times 98.5 \mathrm{~mm} \times 1.57 \mathrm{~mm}$, having a relative permittivity of 2.2. The dimension of each patch element is $41.08 \mathrm{~mm}$ $\times 41.08 \mathrm{~mm}$, and the length and width of the feed are $24.05 \mathrm{~mm}$ and $0.72 \mathrm{~mm}$, respectively. Figure 4 (a) shows the schematic of a microstrip patch antenna array. The distance between the patch elements is $59.74 \mathrm{~mm}(<\lambda / 2)$. Figure 4(b) shows the return loss of the antenna array. 


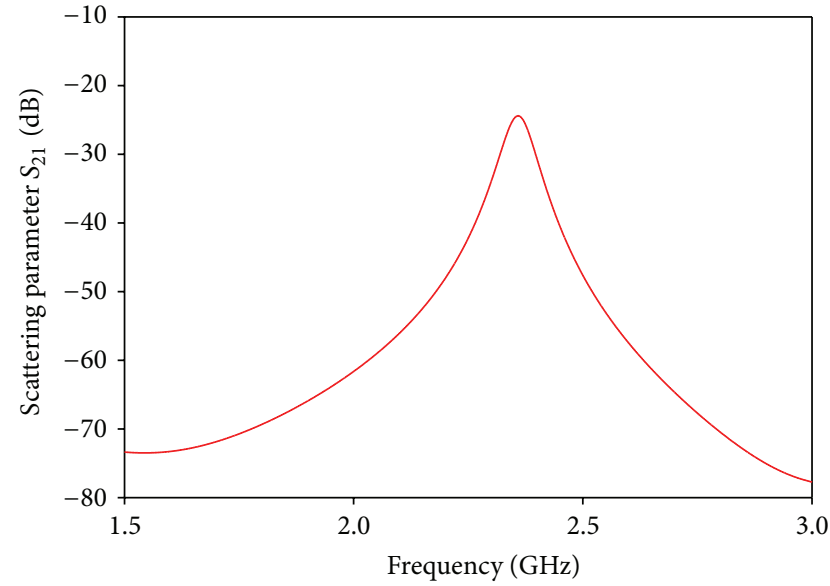

_ Microstrip antenna array without SSRR array

(a)

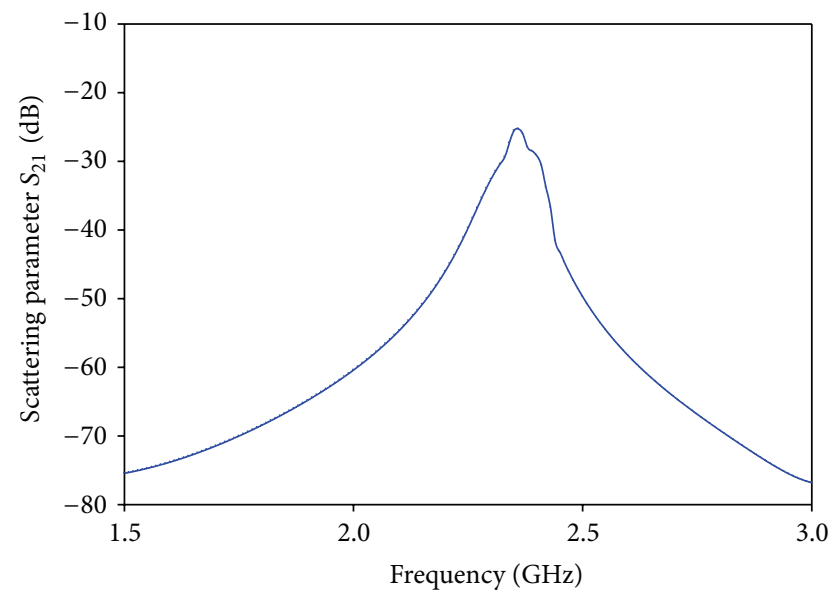

_ Microstrip antenna array with SSRR array at position 2

(c)

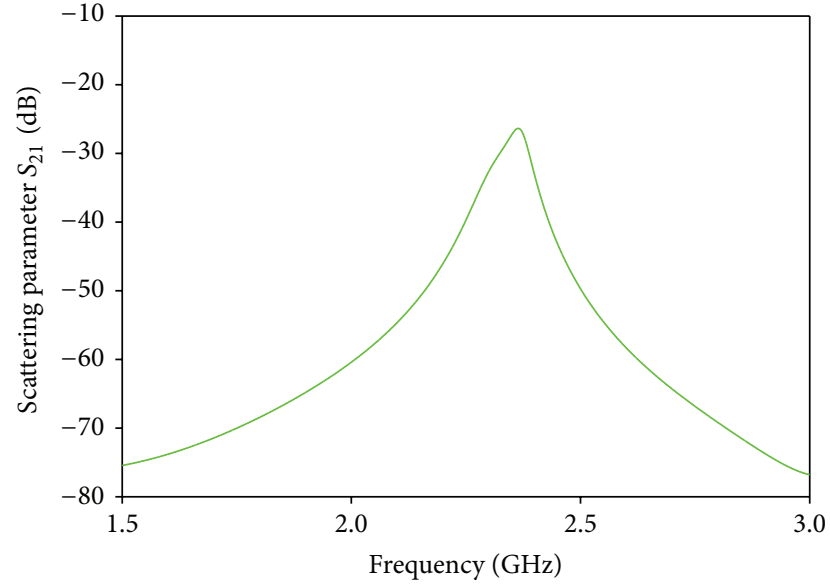

— Microstrip antenna array with SSRR array at position 1

(b)

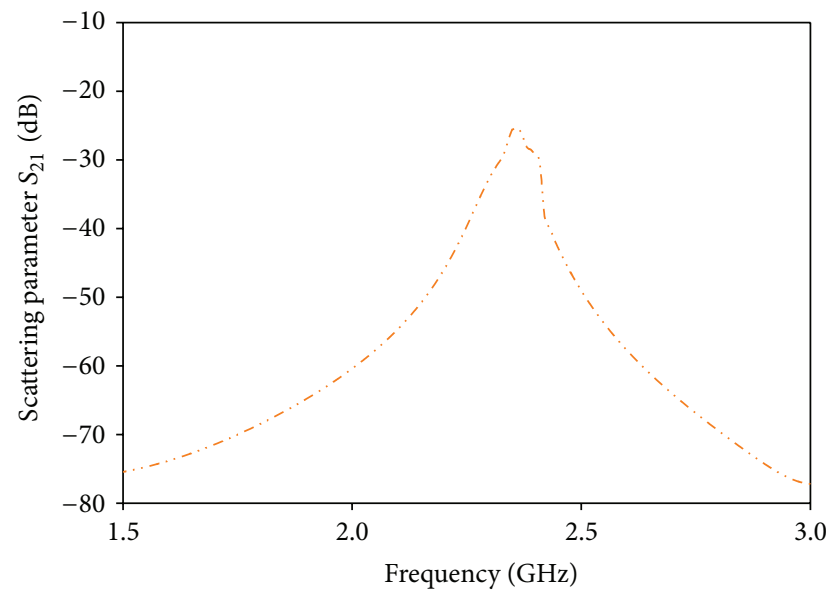

Microstrip antenna array with SSRR array at position 3

(d)

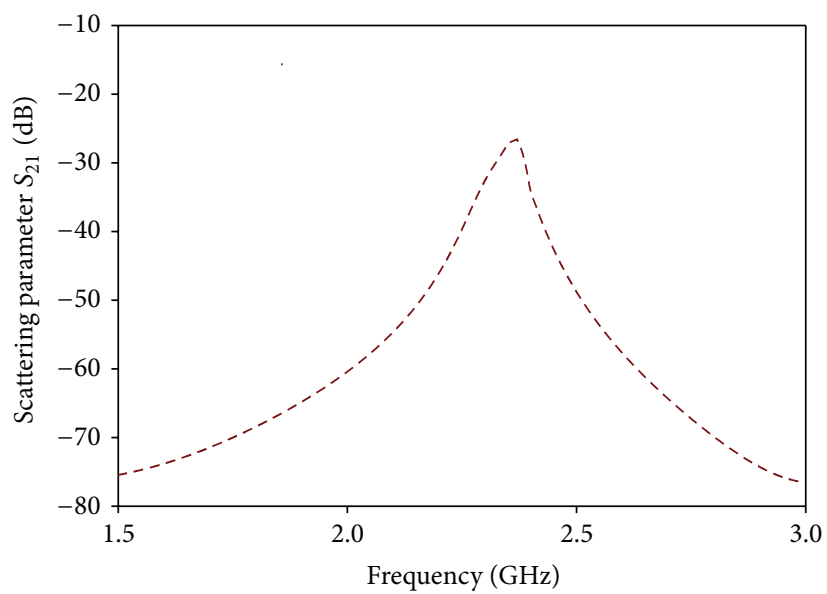

- - - Microstrip antenna array with SSRR array at position 4

(e)

FIGURE 6: (a) Mutual coupling of the antenna array without metamaterial SSRR array, (b) mutual coupling of the antenna array with SSRR array at position 1, (c) mutual coupling of the antenna array with SSRR array at position 2, (d) mutual coupling of the antenna array with SSRR array at position 3, and (e) mutual coupling of the antenna array with SSRR array at position 4 . 
TABLE 1: Comparison of PSO and BFO for structural optimization of double ring square SRR at a particular desired frequency.

\begin{tabular}{lccccc}
\hline $\begin{array}{l}\text { Optimization } \\
\text { techniques }\end{array}$ & Accuracy $\left(f_{\text {err }}\right)$ & $\begin{array}{c}\text { Gap between } \\
\text { rings } d(\text { in } \mathrm{mm})\end{array}$ & $\begin{array}{c}\text { Width of rings } \\
w \text { (in mm) }\end{array}$ & $\begin{array}{c}\text { Length } a \\
\text { (in mm) }\end{array}$ & $\begin{array}{c}\text { Execution time } \\
\text { (in seconds) }\end{array}$ \\
\hline BFO & 0.0071 & 0.30 & 0.21 & 4.90 & 10.51 \\
PSO & 0.000002 & 0.14 & 0.38 & 4.22 & 1.02 \\
\hline
\end{tabular}

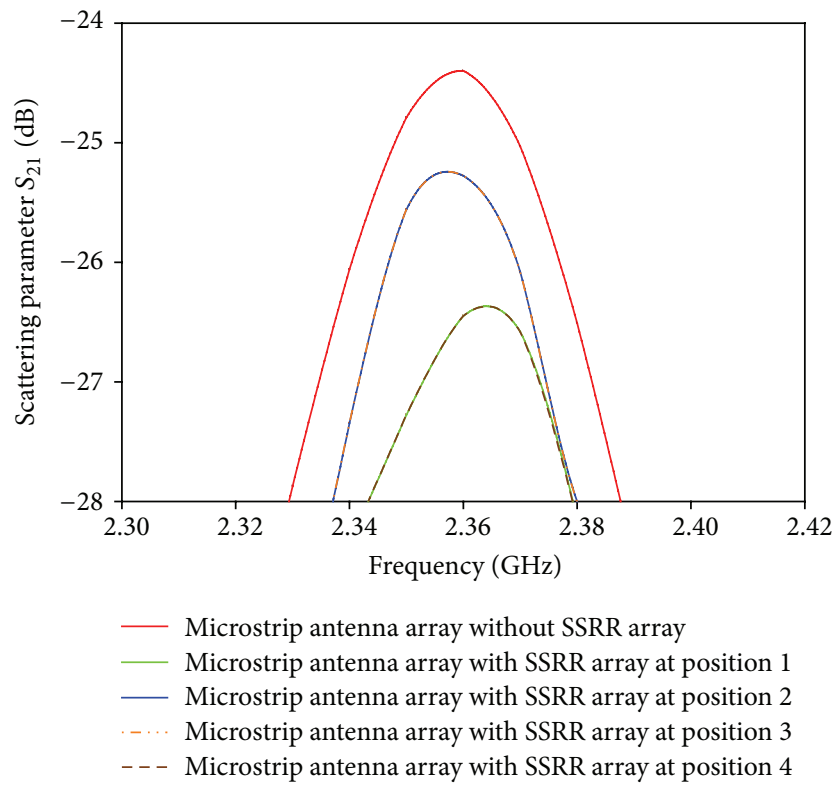

FIGURE 7: Mutual coupling of the antenna array without and with metamaterial SSRR.

\section{Mutual Coupling Effects after Loading SSRR Array}

An array of single ring SSRR is used as a metamaterial structure, which is placed over the antenna array as shown in Figure 5(a). The position of the SSRR array is changed to study the effects on mutual coupling in the antenna array. Four different positions of the SSRR array are shown from Figures 5(a) to 5(d). In Figures 5(a) and 5(d), the SSRR array is placed over the antenna array at a distance of $3.9 \mathrm{~mm}(0.0307 \lambda)$ from the patch element, so that the mutual coupling is reduced by $2.1 \mathrm{~dB}$. Similarly, the mutual coupling is reduced by $1.1 \mathrm{~dB}$ if the SSRR array is placed over the antenna array at a distance of $14.9 \mathrm{~mm}(0.1172 \lambda)$ from the patch element as shown in Figures 5(b) and 5(c). From Figures 6 and 7 it is seen that as the distance between the SSRR array and the patch element increases, mutual coupling increases. Due to symmetry in the feed, the results for the positions 1 and 4 and positions 2 and 3 are identical. By varying the position of the SSRR array closer to or farther from the antenna, it is seen that mutual coupling can be reduced as compared to the antenna array alone.

From the simulation results, we see that the mutual coupling for the antenna array is $-24.4 \mathrm{~dB}$. By keeping an array of SSRR in the position 1 or position 4 (i.e., near the patch element), the mutual coupling is reduced to $-26.5 \mathrm{~dB}$.
By changing the position of the array to positions 3 and 4, mutual coupling is $-25.3 \mathrm{~dB}$. It is seen that the variation in the position of the SSRR array influences mutual coupling. Overall mutual coupling is reduced by $2.1 \mathrm{~dB}$ for positions 1 and 4 .

\section{Conclusion}

PSO is used as an optimization tool for design and optimization of the square split ring resonator (SSRR), which exhibits metamaterial characteristics. The optimized SSRR is used for reduction of mutual coupling in microstrip antenna array designed for wireless applications. It is shown that metamaterial structures, if optimized properly for desired resonant frequency by soft computing techniques such as the PSO, have the potential of yielding excellent features with enhanced performance without resorting to complicated design procedures.

\section{References}

[1] J. A. Bossard, S. Yun, D. H. Werner, and T. S. Mayer, "Synthesizing low loss negative index metamaterial stacks for the midinfrared using genetic algorithms," Optics Express, vol. 17, no. 17, pp. 14771-14779, 2009.

[2] P. N. Fletcher, M. Dean, and A. R. Nix, "Mutual coupling in multi-element array antennas and its influence on MIMO channel capacity," Electronics Letters, vol. 39, no. 4, pp. 342-344, 2003.

[3] E. Rajo-Iglesias, Ó. Quevedo-Teruel, and L. Inclán-Sánchez, "Mutual coupling reduction in patch antenna arrays by using a planar EBG structure and a multilayer dielectric substrate," IEEE Transactions on Antennas and Propagation, vol. 56, no. 6, pp. 1648-1655, 2008.

[4] J. S. R. Jang, C. T. Sun, and E. Mizutani, Neuro-Fuzzy and Soft Computing, Prentice Hall, Upper Saddle River, NJ, USA, 1997.

[5] J. H. Holland, Adaptation in Natural and Artificial Systems, University of Michigan Press, Ann Harbor, Mich, USA, 1975.

[6] J. Kennedy and R. Eberhart, "Particle swarm optimization," in Proceedings of the IEEE International Conference on Neural Networks. Part 1, pp. 1942-1948, December 1995.

[7] K. M. Passino, "Biomimicry of bacterial foraging for distributed optimization and control," IEEE Control Systems Magazine, vol. 22, no. 3, pp. 52-67, 2002.

[8] F. Bilotti, A. Toscano, and L. Vegni, "Design of spiral and multiple split-ring resonators for the realization of miniaturized metamaterial samples," IEEE Transactions on Antennas and Propagation, vol. 55, no. 8, pp. 2258-2267, 2007.

[9] S. Mukherjee, R. Chaudhuri, and C. Saha, "Square split ring resonator: a new approach in estimation of resonant frequency," in Proceedings of the 12th Symposium on Antennas and Propagation (APSYM '10), pp. 146-150, December 2010. 
[10] N. Patel, Theory, simulation, fabrication and testing of double negative and epsilon near zero metamaterials for microwave applications [M.S. thesis], California Polytechnic State University, San Luis Obispo, Calif, USA, 2008.

[11] O. Luukkonen, S. I. Maslovski, and S. A. Tretyakov, "A stepwise Nicolson-Ross-Weir-based material parameter extraction method," IEEE Antennas and Wireless Propagation Letters, vol. 10, pp. 1295-1298, 2011. 


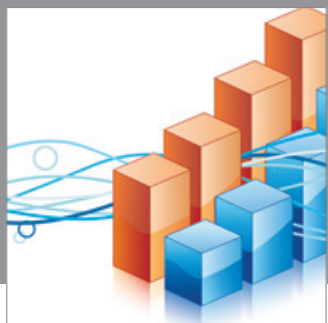

Advances in

Operations Research

mansans

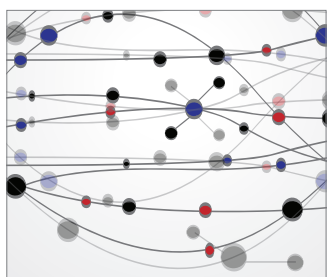

The Scientific World Journal
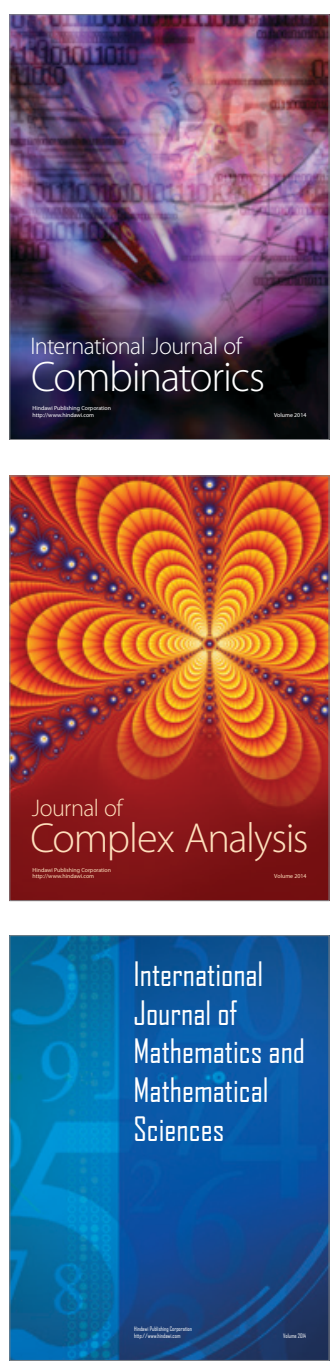
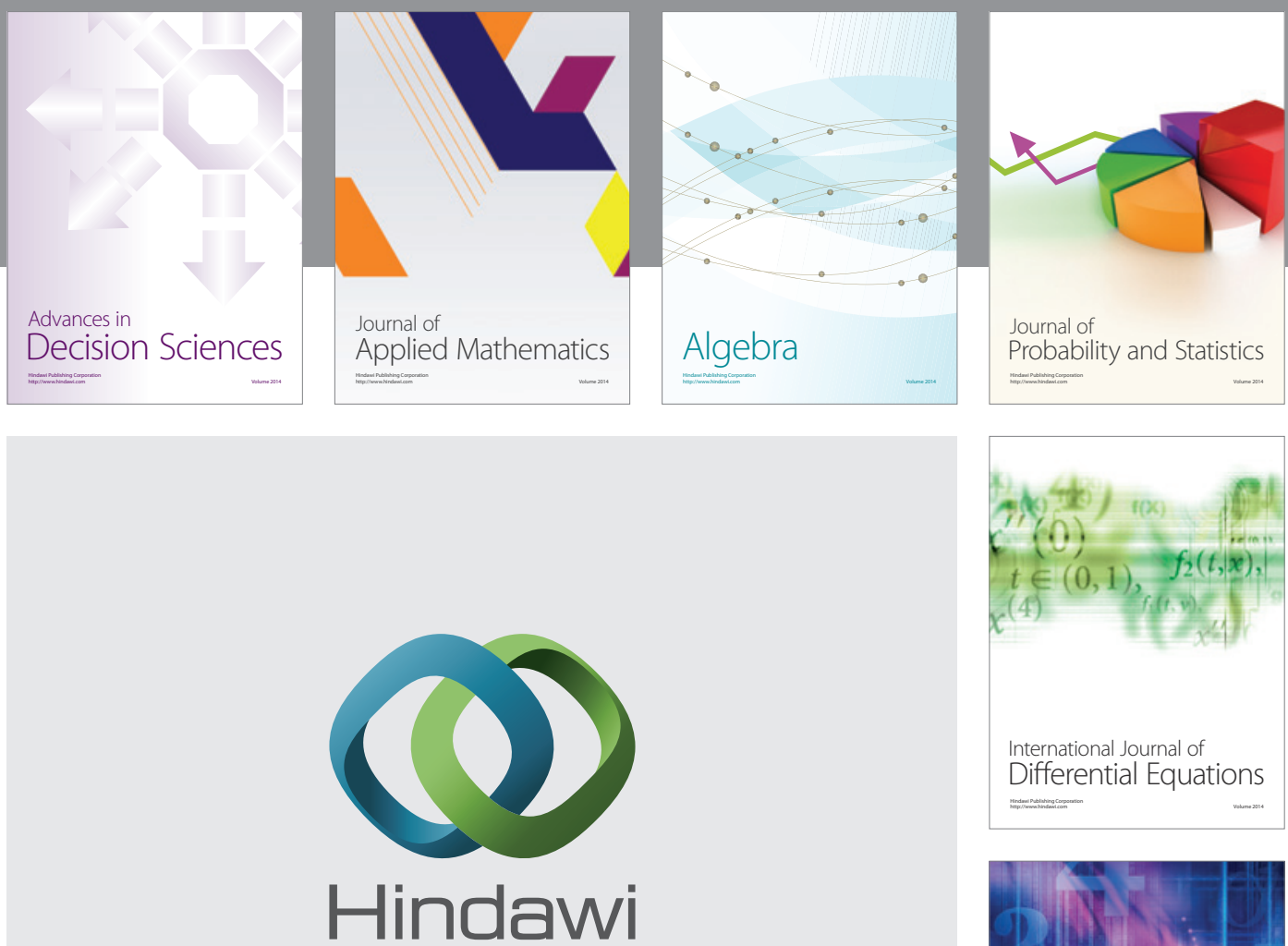

Submit your manuscripts at http://www.hindawi.com
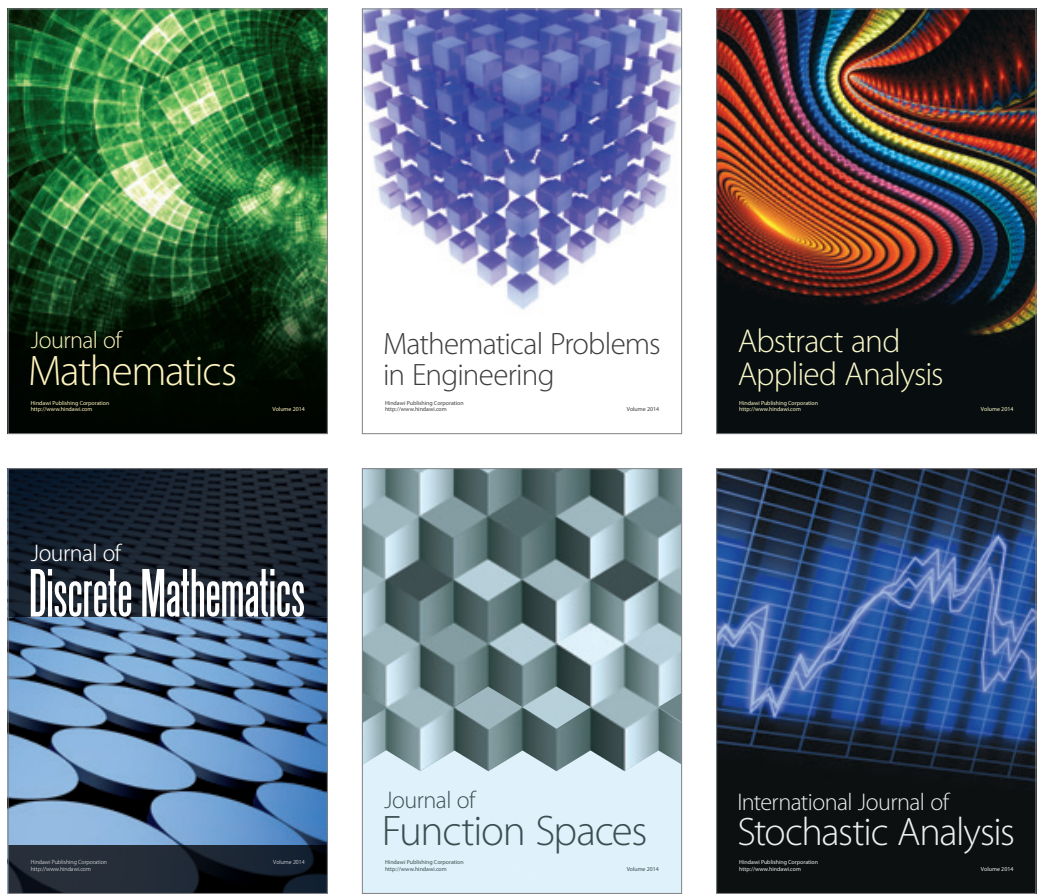

Journal of

Function Spaces



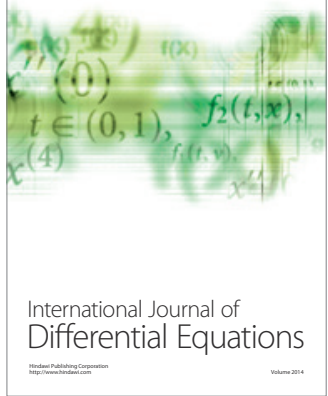
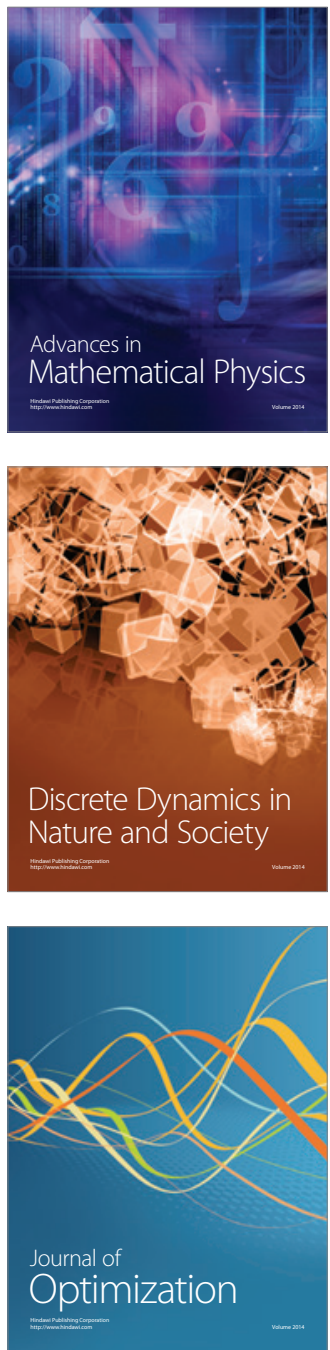